05;15

\title{
Экспериментальное определение поля радиальных перемещений в шейке образцов после испытания на растяжение
}

\author{
() А.А. Быков, Н.В. Буртелова, В.С. Молчанов
}

Московский фризико-технический институт (Государственный университет), Долгопрудный, Московская обл., Россия E-mail: Aleks-Bykov@yandex.ru

Поступило в Редакцию 10 сентября 2018 г.

Представлены результаты исследования полей радиальных перемещений, возникающих при растяжении цилиндрических образцов до их разделения на части. Объектом исследования служила проволока из нержавеющей стали марки св-07X16Н6, содержащая нитевидные дефекты, ориентированные вдоль оси образца. С применением метода рентгеновской томографии шейки образцов определены координаты точек дефектов в образце и с их помощью восстановлено поле перемещений. Полученная зависимость представлена в виде аппроксимационной формулы.

DOI: 10.21883/PJTF.2019.03.47264.17522

Процесс пластических деформаций активно изучается с начала XX века, за это время накоплен значительный экспериментальный материал. На его основе проверяются теории процессов пластического деформирования, подбираются константы материалов, однако для повышения качества подобной работы и точности моделирования необходимо развитие экспериментальных методик. Целью данного исследования является разработка методики получения формульной зависимости радиальных перемещений в шейке цилиндрического образца на основе метода рентгеновской томографии и ее применение к образцам из металлической проволоки.

Впервые метод рентгеновской томографии был применен в 1974 г. по схеме Хаунсфилда и Мак-Кормака и с тех пор принципиально не изменился. В результате его применения восстанавливается эффективный коэффициент рассеяния/поглощения рентгеновского излучения в пространстве. Анализируя полученный результат, можно выделить области, которые заняты одним материалом, или границы между ними. Данная возможность активно применяется для решения задач прочности $[1,2]$.

В настоящее время для восстановления поля перемещений активно используются два метода: VIC-3D [3] и метод спекл-интерферометрии [4]. Методы дают возможность восстанавливать векторы перемещений только внешней поверхности образца и не позволяют определить перемещение внутренних точек. Существуют работы, аналогичные [5], где авторы исследовали образцы с расположенными внутри прочными частицами, легко обнаруживаемыми методом рентгеновской томографии, и определяли относительные перемещения данных частиц. Однако, во-первых, количество таких частиц мало, а во-вторых, из-за их наличия материал можно считать композитным.

В настоящей работе для восстановления поля перемещений используются обнаруживаемые дефекты изготовления, относительная объемная доля которых в образцах значительно меньше процента, что указывает на фактическую однородность исследуемого материала и позво- ляет любому исследователю использовать полученные результаты для определения параметров материала в той или иной модели пластического течения и разрушения.

Экспериментальная методика состоит из четырех этапов. На первом этапе проводятся томографические исследования образцов до и после растяжения. В качестве примера на рис. 1, $a$ приведен поперечный срез томографического изображения стальной проволоки марки св-07X16H6 (C - 0.7\%, Cr - 1.6\%, Ni - 0.6\%) до растяжения. На изображении можно обнаружить некоторое количество вытянутых вдоль оси дефектов. На рис. $1, b$ показан продольный срез томографического изображения шейки, образовавшейся в результате растяжения указанного образца. На нем видны нитеобразные дефекты.

При сличении двух изображении можно сделать следующее предположение (далее „предположение“). При изготовлении в проволоке образуются вытянутые вдоль оси объемные дефекты, которые в процессе испытания сохраняются (не исчезают, не затекают). Если дефект достаточно длинный, то после разрушения образца он может начинаться в цилиндрической части, переходить в шейку с искривлением и заканчиваться в месте разрыва (см. рис. 1, $c$, длинный дефект показан схематически, остальные обведены контрастными линиями). Длина стрелки на рисунке равна радиальному перемещению точки длинного дефекта в процессе пластической деформации. Таким дефектом также можно считать боковую поверхность образца.

Второй этап методики заключается в определении координат дефектов. Координаты дефектов на томографическом изображении определяются в цилиндрических координатах относительно продольной оси образца. Затем координаты проецируются на положительную четверть плоскости, где абсциссой служит ось образца, а ординатой - его радиус. На рис. 2 показан результат для одного образца. Видно, что дефектов в шейке довольно много, но ни один из них не начинается в цилиндри- 

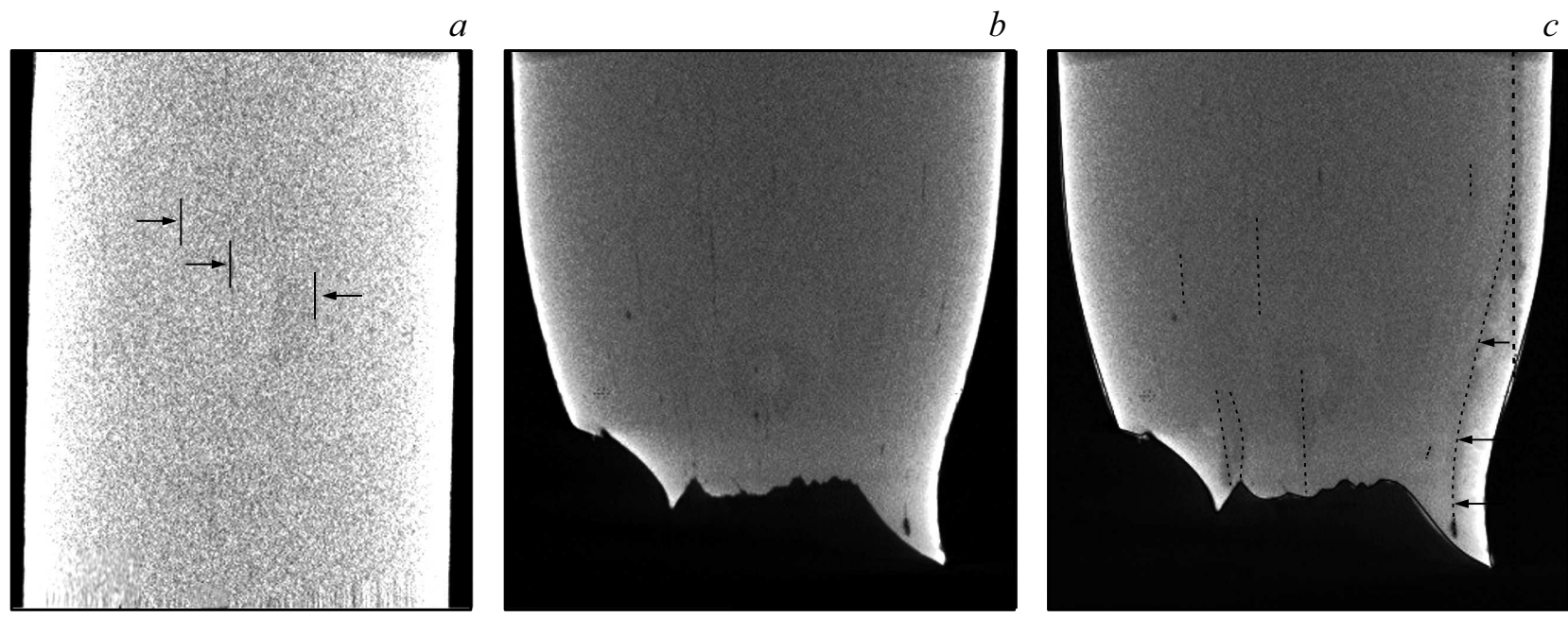

Рис. 1. Томографическое изображение образца. $a-$ сечение трехмерного изображения образца до испытания на растяжение (стрелками показаны дефекты); $b$ - сечение трехмерного изображения шейки образца после испытания; $c$ - сечение образца с выделенными линиями дефектов (пунктирная линия - положение дефекта в образце, стрелками показаны радиальные перемещения точек дефекта).

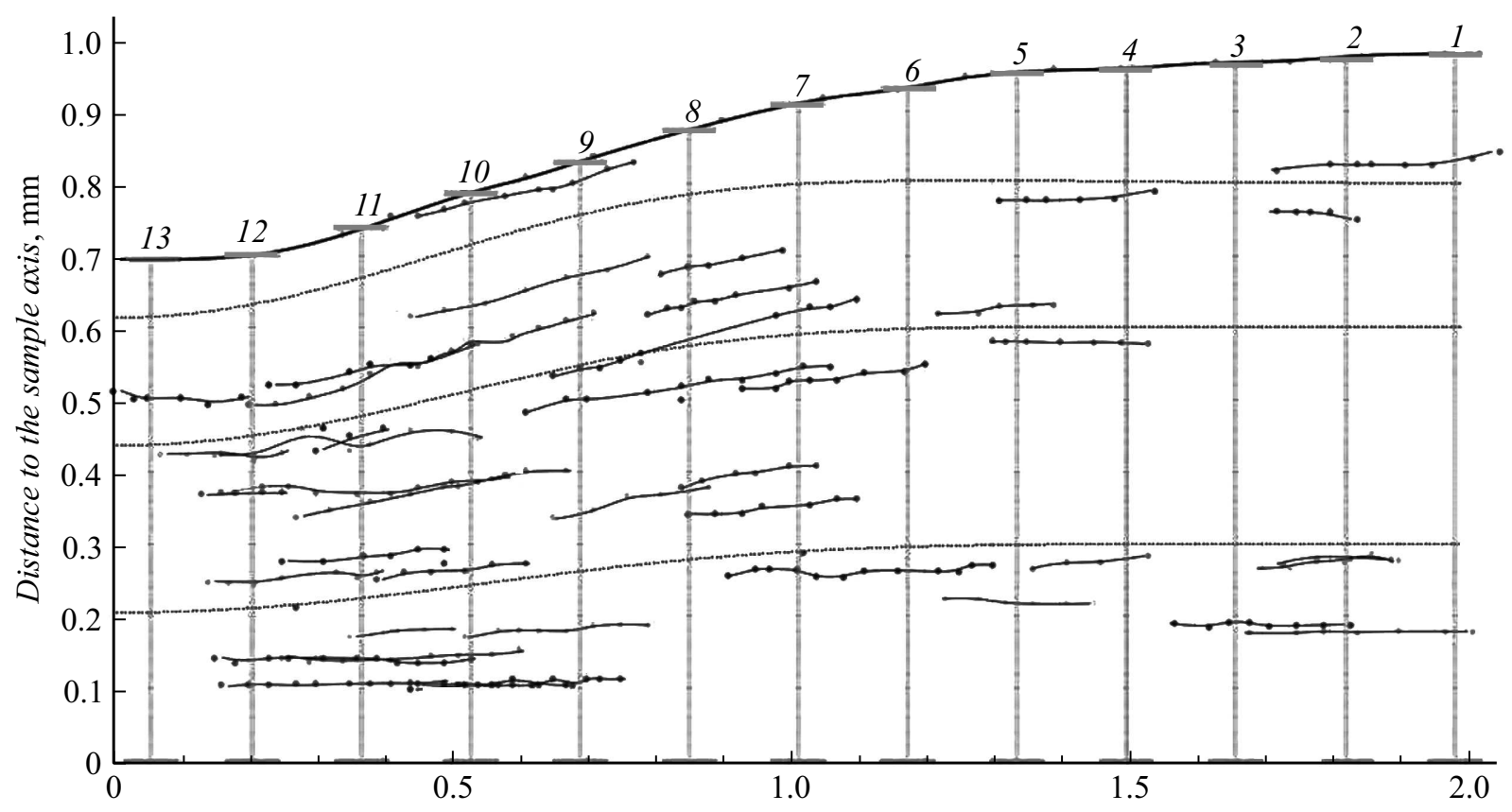

Distance along the sample, $\mathrm{mm}$

Рис. 2. Линии дефектов в шейке образца. Линии, показанные пунктиром, восстановлены по аппроксимационной формуле и до испытания были параллельны оси образца. Цифры поясняются в тексте.

ческой части и не заканчивается в месте разрушения шейки.

На третьем этапе образец делится на ряд сечений таким образом, чтобы бо́льшая часть дефектов пересекала несколько таких сечений (см. рис. 2, сечения пронумерованы в верхней части рисунка). Тогда возможно восстановление поля перемещений в следующем порядке. Выбирается сечение в цилиндрической части образца, перемещение точек которого можно считать равным нулю. Затем, используя „предположение“, можно определить перемещения точек в следующем сечении по дефектам, которые одновременно их пересекают. Во втором сечении по полученным координатам проводится аппроксимация перемещений в зависимости от расстояния до оси образца $r$.

В работе было использовано программное обеспечение OriginPro 9.0, которое позволяет аппроксимировать экспериментальные точки функциями различного вида. 
В результате было установлено, что для каждого сечения достаточно определять поле радиальных перемещений как полином второго порядка, такая аппроксимационная функция дает наименьшую погрешность из всех функций, которые позволяет применить OriginPro.

Далее выбираются второе сечение и дефекты, пересекающие данное сечение и первое, определяется разность перемещений точек, принадлежащих одному дефекту, но находящихся в различных сечениях. Это соответствует относительному перемещению данных точек, а в качестве перемещения точки во втором сечении можно принять сумму относительного перемещения и уже определенного на предыдущем шаге перемещения соответствующей точки в первом сечении. Затем проводится аппроксимация поля радиальных перемещений полиномом второй степени.

Указанный алгоритм шаг за шагом применяется для последовательно расположенных сечений, пока не будут определены перемещения достаточно большого количества точек в образце.

На четвертом этапе проводится аппроксимация полученной зависимости радиальных перемещений точек в зависимости от двух координат: $r$ и $z$. При этом она должна удовлетворять некоторым требованиям. Математически их можно сформулировать следующим образом:

$$
\left.U_{r}\right|_{r=0}=0, \quad \partial U_{r} /\left.\partial z\right|_{z=0}=0, \quad \partial U_{r} /\left.\partial z\right|_{z \rightarrow+\infty}=0 .
$$

Первое условие следует из того, что на оси образца не наблюдается нарушения сплошности и в процессе деформации точки на оси перемещаются строго вдоль нее. Второе условие возникает из ожидания четности (симметричности) радиальных перемещений относительно плоскости симметрии образца, третье следует из однородности полей перемещений в цилиндрической части вне шейки.

При аппроксимации было определено, что перемещения в каждом сечении, перпендикулярном оси образца, хорошо описываются полиномом второго порядка без постоянной части, поэтому аппроксимационная функция была выбрана в следующем виде:

$$
U(r, z)=-0.4 \exp \left(-z^{2} / 0.59\right) r^{2}+0.55 \exp \left(-2.2 z^{2}\right) r^{2} .
$$

Относительная погрешность определения коэффициентов в показателях экспонент составила 20\%. Все величины, входящие в формулу, указаны в миллиметрах.

В процессе работы испытания образцов проводились на разрывной машине Com-Ten, их первоначальный диаметр составлял $1.93 \mathrm{~mm}$ с точностью $0.02 \mathrm{~mm}$, длина между захватами $238 \mathrm{~mm}$. Полученная диаграмма нагружения приведена на рис. 3.

Таким образом, в работе предложена методика определения радиальных перемещений в области шейки образца после разрушения и результат ее применения к пяти образцам, изготовленным из нержавеющей стали.

Необходимо отметить, что методика позволяет определить перемещения относительно координат, связанных

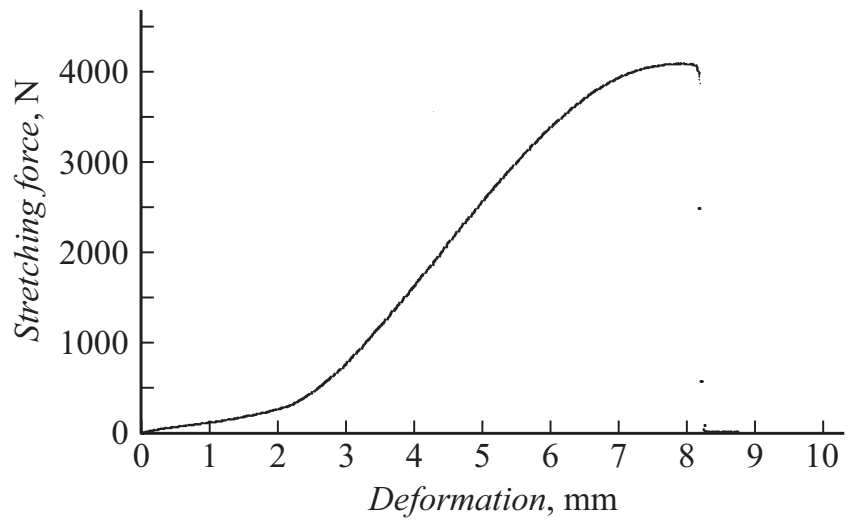

Рис. 3. Диаграмма нагружения образца.

с конечным состоянием образца. Как правило, при расчетах или экспериментальной работе определяется поле перемещений относительно координат, связанных с начальным состоянием образцов, и при использовании приведенных результатов необходимо это учитывать.

Для применения методики необходимо, чтобы до нагружения образцы содержали длинные и легко обнаруживаемые дефекты, параллельные оси и относительно равномерно распределенные по объему.

В области шейки образца ожидается, что зависимости, приведенные на рис. 3, должны быть монотонно возрастающими, но при внимательном рассмотрении некоторые линии не удовлетворяют указанному условию. Объяснить это можно тем, что в образцах небольшая часть дефектов не параллельна оси.

Для анализа полученных результатов нами приведены первичные экспериментальные результаты, показанные на рис. 2 и 3. По ним возможно построение собственной аппроксимационной функции и определение постоянных для указанного материала для различных теорий пластичности.

\section{Список литературы}

[1] Kinney J.H., Nichols M.C. // Annu. Rev. Mater. Sci. 1992. V. 22. P. 121-152.

[2] Marrow T.J., Buffiere J.-Y., Withers P.J., Johnson G., Engelberg D. // Int. J. Fatique. 2004. V. 26. P. 717-125.

[3] Третьякова Т.В. // Вычислительная механика сплошных сред. 2014. Т. 77. № 2. С. 162-171.

[4] Баранникова С.А., Надежкин М.В., Лунев А.Г., Горбатенко В.В., Зуев Л.Б. // Письма в ЖТФ. 2014. Т. 40. В. 5. С. 5158.

[5] Withers P.J., Preuss M. //Annu. Rev. Mater. Res. 2012. V. 42. P. $81-103$. 